\title{
Optimization of Sensor Locations and Sensitivity Analysis for Engine Health Monitoring Using Minimum Interference Algorithms
}

\author{
Paul Cotae, Sireesha Yalamnchili, C. L. Philip Chen, and Arturo Ayon \\ Department of Electrical and Computer Engineering, Wireless Sensor Network Laboratory, \\ University of Texas at San Antonio, 6900 North Loop 1604 West, San Antonio, TX 78249-0669, USA
}

Correspondence should be addressed to Paul Cotae, paul.cotae@utsa.edu

Received 10 June 2007; Revised 20 August 2007; Accepted 8 October 2007

Recommended by Erchin Serpedin

\begin{abstract}
The global optimization of sensor locations and a sensitivity analysis based on the minimization of interferences due to wireless communications between sensors are studied in the presence of additive white Gaussian noise (AWGN). We used a Gram matrix approach for robust determination of sensor locations by minimizing the interferences (maximizing the signal strength) among sensors for engine health monitoring systems. In order to solve the problem of optimum placement, an iterative algorithm for maximizing the determinant of the Gram matrix is proposed and implemented. The sensitivity criterion proposed in this paper is the spectral number of the Frobenius norm of the Gram matrix associated with sensor readings. We derived the necessary conditions under which the number of sensors and the optimal sensor locations will remain unchanged when the data measured for sensitivity analysis is affected by AWGN. Our theoretical results are verified by simulations providing details concerning numerical implementations.
\end{abstract}

Copyright (C) 2008 Paul Cotae et al. This is an open access article distributed under the Creative Commons Attribution License, which permits unrestricted use, distribution, and reproduction in any medium, provided the original work is properly cited.

\section{INTRODUCTION}

The problem of sensor location is crucial for system identification, control, and damage detection which requires accurate measurement of the responses of the system [1-8]. The measured data are always inaccurate because of the existence of parameter variation or noise. Taking the cost of sensors into account, it is not economical to install sensors in every part of system. In such cases, a practical question that naturally arises is how to select a set with a minimum number of sensor locations from all possibilities, such that the data collected may provide the greatest opportunities for the detection of failures [9-12]. This problem has been widely investigated in the last decade and many methods such as the minimal energy principle, mode shape independent principle, Fisher information matrix, effective interference method, and so forth have been developed for the determination of sensor locations in many publications $[5,8,11]$. However, these placement optimization criteria focused on maximizing either the controllability or the observability of the system, assuming that the sensor readings are not affected by wireless interferences [13].
In this paper, we address the problem of selecting the optimal location of sensors, such that the resulting sensor readings provide maximum information about the condition of the structure as specified by values of the model parameters. We assume that each sensor has wireless communication capabilities, in that each sensor reading is characterized in terms of signal-to-noise ratio. Optimal and effective deployment of sensors in health engine monitoring is difficult and critical because the number of sensors is usually limited and their locations are fixed, once installed. We present a methodology for the optimal placement of sensors to be used in the diagnosis of engine failure considering all the interferences from different locations affecting all the sensors in the system [14-16].

Optimal sensor location is a combinatorial problem where one seeks to place $N_{S}$ sensors out of $M$ potential locations. The test of all possibilities is rarely realistic; thus one can consider sets of $N$ sensors simultaneously (simulated annealing, genetic searches $[13,17]$ ), or sequential approaches where one removes or adds one sensor at a time. In this paper, we start with a large number of candidate locations $M$ by eliminating the least effective sensor in each step of iteration 
until a given number of sensors are left. The optimality criterion used is the optimization of the determinant of Gram matrix $\mathbf{G}=\mathbf{D}^{\mathrm{T}} \mathbf{D}$ [14], where the matrix $\mathbf{D}$ is of the dimension $L \times N$. It is assumed that each sensor reading is a onedimensional vector of dimension $L \leq N<M$. The objective of this paper is to find the optimum number of sensors $N$ out of $M$ possible locations and should not be confused with the dimensionality reduction, where the goal is to reduce the value of length of vector observability $P[9,18,19]$.

The objective function, the optimization of Gram matrix determinant, may have multiple local optima and saddle points, and hence any local search might stagnate at a suboptimal solution. Following the work in [14], the proposed method is convergent, having a unique global optimum. Previous work, addressing the issue of optimality location of a given number of sensors, has been carried out by several investigators. The criteria used are trace norm [5], determinant norm [20], and extremal eigenvalue of the Fisher information matrix [7]. The approach based on the Gram matrix was also considered in another context in [16] where a metric related to all interferences in the wireless network was introduced, namely, the total weighted squared correlation. This paper is organized as follows. In Section 2, we introduce the system model, and the problem formulation for optimum placement is presented in Section 3. The sensitivity analysis is derived in Section 4. The computational procedure for the optimal sensor placement method is given in Section 5 . The numerical results are presented in Section 6 and conclusions are drawn in Section 7.

\section{SYSTEM MODEL}

Consider a wireless sensor network composed of $N$ sensors distributed at known spatial locations, each data sensor reading vector, $1 \leq i \leq N$, is in transpose form, having a dimension $P \times 1$, and $P \leq N$ :

$$
\mathbf{d}^{(i)}=\left\{d_{1}^{(i)}, d_{2}^{(i)}, \ldots, d_{P}^{(i)}\right\}^{T}, \quad i=1,2, \ldots, N .
$$

The vector from measurements denoted by $\mathbf{y}$ is composed by data from each of $N$ sensors $\mathbf{y}=\left\{y_{1}, y_{2}, \ldots, y_{N}\right\}^{T}$. The individual contribution of each sensor is denoted by $q_{i}(i=$ $1,2, \ldots, N)$ and it is included in the vector $\mathbf{q}=\left\{q_{1}, q_{2}, \ldots\right.$, $\left.q_{N}\right\}^{T}$. The mathematical model used for sensor measurements vector $\mathbf{y}^{1}$ is given by the following matrix equation:

$$
\mathbf{y}=q_{1} \mathbf{d}^{(1)}+q_{2} \mathbf{d}^{(2)}+\cdots+q_{N} \mathbf{d}^{(N)}+\mathbf{w}=\mathbf{D q}+\mathbf{w},
$$

where $\mathbf{D}$ is a $P \times N$ the matrix having the designated pattern vectors $\mathbf{d}^{(i)}$ as columns, $\mathbf{w}$ is a vector representing the measurement noise and all other effects due to the interferences; it is assumed to have a Gaussian distribution with zero mean and the covariance matrix $\boldsymbol{\Sigma}=\boldsymbol{\sigma}_{\mathrm{W}}^{2} \mathbf{I}_{\mathrm{N}}$, where $\mathbf{I}_{\mathbf{N}}$ is the unity matrix of order $N$. We assume that sensor readings are

\footnotetext{
${ }^{1}$ We will assume $y=[y_{1}, \ldots, y_{P}, \underbrace{0, \ldots, 0}_{N-P}]$ if no noise.
}

affected by independent noise with equal variances and the variance of noise in the system can be estimated from measurements. Using the Gram matrix $\mathbf{G}=\mathbf{D}^{\mathrm{T}} \mathbf{D}$ in the leastsquares method ${ }^{2}$ [21], an estimate of $\mathbf{q}$ in the mathematical model (2) is obtained $\mathrm{as}^{3}$

$$
\widehat{\mathbf{q}}=\mathbf{G}^{-1} \mathbf{D}^{\mathrm{T}} \mathbf{y}
$$

The accuracy of estimation for $\hat{\mathbf{q}}$ may be evaluated to be the error of estimation which is the expected value of the quantity $(\mathbf{q}-\hat{\mathbf{q}})(\mathbf{q}-\hat{\mathbf{q}})^{T}$ being related to the inverse of the Fisher information matrix $\mathbf{F}$ [5] as follows:

$$
E\left[(\mathbf{q}-\hat{\mathbf{q}})(\mathbf{q}-\hat{\mathbf{q}})^{T}\right]=\mathbf{F}^{-1}
$$

Several scalar performance measures related to the information matrix $\mathbf{F}$ were studied in the past. The most general one is the following criterion based on the trace of the matrix:

$$
m_{s}=\left(\frac{1}{N} \operatorname{trace}\left(\mathbf{F}^{s}\right)\right)^{1 / s},
$$

where the parameters $s \leq 0$ and $N$ represent the number of sensors. By particularizing $s$, then the following widely used criteria are obtained:

(a) determinant norm: $\lim _{s \rightarrow 0} m_{s}=|F|^{1 / N}$,

(b) trace norm $m_{-1}=N / \operatorname{trace}\left(F^{-1}\right)$,

(c) extremal eigenvalue $m_{-\infty}=\lambda_{\min }(F)$.

Different from previous criteria used so far for optimal sensor location problems, in this paper we will use the Gram matrix approach originally introduced in the context of interference avoidance in [14]. The interference metric total weighted squared correlation (TWSC) [16] is expressed in terms of the Frobenius norm associated to the Gram matrix, and it is given by the sum of its squared eigenvalues, while the determinant of the Gram matrix is the product of its eigenvalues. By using the arithmetic-geometric mean inequality [22], the superiority of choosing the Gram matrix approach becomes evident, since the determinant norm, trace norm, extremal eigenvalues criteria, and (5) are particular cases, and furthermore the Gram matrix approach provides a tighter lower bound. When the arithmetic-geometric

\footnotetext{
2 The least-squares (LS) estimator is asymptotically unbiased. The Cramer Rao bound (CRB) provides a lower bound on the error variance in estimating the model parameters for any unbiased estimator of these parameters. Assuming that the observed field is a Gaussian random field, the asymptotic error covariance matrix of the best LS attains the CRB even for the modest dimensions of the observed field and low signal-tonoise ratios. We conjecture that our results hold when the data dimension becomes larger; the number of deployed sensors is larger as in the real applications for wireless sensors networks.

3 Two different forms of Gram matrices are associated to the wireless sensor networks. For an overloaded network where $P \leq N$, and considered here, we will use $\mathbf{G}=\mathbf{D}^{\mathrm{T}} \mathbf{D}$ of dimension $N \times N$. This is in contrast with underloaded networks $(P>N)$ where the dimensionality of Gram matrix $\mathbf{G}=\mathbf{D D}^{\mathrm{T}}$ is $P \times P$. Equation (3) is valid only if the inverse of the Gram matrix $\mathbf{G}=\mathbf{D}^{\mathrm{T}} \mathbf{D}$ exists. The condition of the inverse of $\mathbf{G}$ is equivalent to the full rank of the Gram matrix, $N$, which in turn is equivalent to the linear independence of vector $\mathbf{d}^{(i)}$ that constitutes $\mathbf{D}$.
} 
mean inequality is applied to the TWSC, the lower bound is again given in terms of the product of its eigenvalues, which in fact is the determinant of Gram matrix.

The Fisher information matrix from (4), by using (3), becomes

$$
\mathbf{F}=\frac{1}{\sigma_{w}^{2}} \mathbf{D}^{T} \mathbf{D}=\frac{1}{\sigma_{w}^{2}} \mathbf{G} .
$$

In order to minimize the error of estimation given by (4) we need to maximize the determinant of the Gram matrix given by (6). Suppose now that sensor reading $i$ is decoded using a linear receiver denoted by $c_{i} .{ }^{4}$ Writing $\mathbf{Y}=\left(y_{1}, y_{2}, \ldots, y_{N}\right)$ as a vector in $R^{N}$, then the received signal can be written as

$$
\mathbf{Y}=\sum_{i=1}^{M} q_{i} \mathbf{d}^{(i)}+\mathbf{W}
$$

where $\mathbf{W}$ is an independent and identically distributed (i.i.d.) Gaussian vector with covariance $\sigma^{2} \mathbf{I}_{N}$ independent of sensor readings. Based on $(7)^{5}$ the signal-to-interference ratio of user $i\left(\mathrm{SIR}_{i}\right)$ is

$$
\operatorname{SIR}_{i}=\frac{\left(\mathbf{c}_{\mathbf{i}}, \mathbf{d}^{(\mathbf{i})}\right)^{2} q_{i}}{\sigma^{2}\left(\mathbf{c}_{\mathbf{i}}, \mathbf{c}_{\mathbf{i}}\right)+\sum_{j \neq i}\left(\mathbf{c}_{\mathbf{i}}, \mathbf{d}^{(\mathbf{j})}\right)^{2} q_{j}}
$$

We say that a set of $M$ sensors is optimum for placement and reliable communication if there is a positive set $\left\{q_{i}\right\}$ and linear receiver structures $\mathbf{c}_{\mathbf{i}}$, such that the signal-to-interference ratio for each user is that $\operatorname{SIR}_{i} \geq \beta, \forall i=1 \cdots M$. Here $\beta>0$ is some fixed SIR requirement of each user that has to be met for satisfactory performance. The optimum receiver $c_{i}$ is one that maximizes $\operatorname{SIR}_{i}$ assuming that the channel state information is known perfectly both at transmitter and receiver. The system model proposed in this paper characterized by (2) and (8) is used for engine health monitoring; no other work is known to the authors focusing on the optimum sensor location based on their ability to communicate with each other in the presence of wireless interference.

\section{PROBLEM FORMULATION}

Fixing the set $\left\{q_{i}\right\}$ and the sensor readings $\mathbf{d}^{(i)}$, for each sensor $1 \leq i \leq M$, let us consider the matrices $\mathbf{D}=$ $\left[\mathbf{d}^{(1)}, \mathbf{d}^{(2)}, \ldots, \mathbf{d}^{(M)}\right]$ and $\mathbf{Q}=\operatorname{diag}\left(q_{1}, q_{2}, \ldots, q_{M}\right)$, respectively, by including all sensors in the system. Now, consider the same matrices when the sensor reading $i$ is deleted from the system: $\mathbf{D}_{-i}=\left[\mathbf{d}^{(1)}, \ldots, \mathbf{d}^{(i-1)}, \mathbf{d}^{(i+1)}, \ldots, \mathbf{d}^{(M)}\right]$ and $\mathbf{Q}_{-i}=\operatorname{diag}\left(q_{1}, \ldots q_{i-1}, q_{i+1} \ldots, q_{M}\right),{ }^{6}$ respectively. Based on

\footnotetext{
${ }^{4}$ We assume that each sensor has wireless communication capabilities.

5 The difference between (7) and (2) consists not only in terms of summation. In (2) we have an estimation problem while in (7) we have a multiuser detection problem where the set $q_{i}$ is not necessarily independent. If the linear receiver structures are independent, the $\mathbf{D}$ matrix becomes a full matrix, and thus (3) simply yields $\hat{q}=\mathbf{D}^{-1} \mathbf{y}$. In addition, in (2) we need to solve $N$ equations for $N$ unknowns $q_{i}(i=1,2, \ldots, N)$.

${ }^{6}$ We will denote the diagonal matrix whose main diagonal entries are the same as those of the matrix $\mathbf{A}$ as $\operatorname{diag}(\mathbf{A})$ and the diagonal matrix whose diagonal entries are formed from vector $\mathbf{b}$ as $\operatorname{diag}(\mathbf{b})$.
}

the above matrices let us consider another two matrices defined as $Z_{-i}=D_{-i} Q_{-i} D_{-i}^{T}+\sigma^{2} I$ and $Z=D Q D^{T}+\sigma^{2} I$. These two matrices are positive definite having the following eigenvalue decomposition [22] for some positive diagonal matrices $\boldsymbol{\Lambda}_{i}$ and $\boldsymbol{\Lambda}: \mathbf{Z}_{-i}=\mathbf{U}_{i} \boldsymbol{\Lambda}_{i} \mathbf{U}_{i}^{T}, \mathbf{Z}=\mathbf{U} \boldsymbol{\Lambda} \mathbf{U}^{T}$, where $\mathbf{U}, \mathbf{U}_{i}$ are unitary matrices. Then using (8) for each sensor $i$, we obtain the following expression:

$$
\begin{aligned}
& \max _{c_{i} \neq 0} \operatorname{SIR}_{i}=\max _{c_{i} \neq 0} \frac{\left(\mathbf{c}_{\mathbf{i}}, \mathbf{d}^{(\mathbf{i})}\right)^{2} q_{i}}{\mathbf{c}_{\mathbf{i}}^{T} \mathbf{Z}_{\mathbf{i}} \mathbf{c}_{\mathbf{i}}} \\
& =q_{i} \max _{x_{i} \neq 0} \frac{\mathbf{x}_{\mathbf{i}}^{T} \boldsymbol{\Lambda}_{i}^{-1 / 2} \mathbf{U}_{i}^{t} \mathbf{d}^{(\mathbf{i})} \mathbf{d}^{(\mathbf{i}) T} \mathbf{U}_{i} \boldsymbol{\Lambda}_{i}^{-1 / 2} \mathbf{x}_{\mathbf{i}}}{\mathbf{x}_{\mathbf{i}}^{T} \mathbf{x}_{\mathbf{i}}}, \\
& \mathbf{x}_{\mathbf{i}}=\boldsymbol{\Lambda}_{i}^{-1 / 2} \mathbf{U}_{i}^{T} \mathbf{c}_{\mathbf{i}}
\end{aligned}
$$

In the above expression the optimal receiver structure is $\mathbf{c}_{i}=$ $\mathbf{Z}^{-1} \mathbf{d}^{(i)}$. By using the following formula:

$$
\left(\mathbf{A}-\mathbf{x} \mathbf{y}^{T}\right)^{-1}=\mathbf{A}^{-1}+\frac{\mathbf{A}^{-1} \mathbf{x} \mathbf{y}^{T} \mathbf{A}^{-1}}{1-\mathbf{y}^{T} \mathbf{A}^{-1} \mathbf{x}},
$$

whenever the terms exist, from (8) to (9), we have successively

$$
\begin{aligned}
\operatorname{SIR}_{i} & =\mathbf{d}^{(i) T} \mathbf{Z}^{-1} \mathbf{d}^{(i)} \mathbf{c}_{i} \\
& =\mathbf{d}^{(i) T}\left(\mathbf{Z}^{-1}-\mathbf{c}_{i} \mathbf{d}^{(i)} \mathbf{d}^{(i) T} \mathbf{Z}^{-1}\right) \mathbf{d}^{(i)} \mathbf{c}_{i} \\
& =\mathbf{d}^{(i) T}\left(\mathbf{Z}^{-1}-\frac{\mathbf{c}_{i} \mathbf{d}^{(i)} \mathbf{d}^{(i) T} \mathbf{Z}^{-1} \mathbf{c}_{i}}{1-\mathbf{d}^{(i) T} \mathbf{Z}^{-1} \mathbf{d}^{(i)} c_{i}}\right) \mathbf{d}^{(i)} \mathbf{c}_{i} \\
& =\frac{\mathbf{d}^{(i) T} \mathbf{Z}^{-1} \mathbf{d}^{(i)} \mathbf{c}_{i}}{1-\mathbf{d}^{(i) T} \mathbf{Z}^{-1} \mathbf{d}^{(i)} \mathbf{c}_{i}} .
\end{aligned}
$$

Here $\mathbf{d}^{(i) T} \mathbf{Z}^{-1} \mathbf{d}^{(i)}$ is strictly less than 1 and thus all the terms above are well defined.

Mathematically the optimization problem of sensor placement in the presence of the interferences can be formulated as follows: given the sensor reading matrix $\mathbf{D}=$ $\left[\mathbf{d}^{(1)}, \mathbf{d}^{(2)}, \ldots, \mathbf{d}^{(M)}\right]$, and SIR requirement $\beta$, for $\forall i, M \geq i \geq$ $N$, find the Gram matrix $\mathbf{G}$ whose determinant is maximum:

$$
\begin{aligned}
\mathbf{G} \in \arg \max \operatorname{det} \mathbf{G} \\
\quad=\left(1-d_{i}^{T} \mathbf{G}_{-i}^{-1} d_{i}\right) \operatorname{det} \mathbf{G}_{-i} \quad \text { such that } \operatorname{SIR}_{i} \geq \beta .
\end{aligned}
$$

Since the Gram matrix is a summation of the contribution of each degree of freedom or sensor location to the mode shape of structural engine health monitoring, maximizing its determinant will lead to the best placement in the presence of interferences. A formal description of our optimization problem can be formulated as follows: let $\mathbf{G}_{-i}=\mathbf{D}_{-i}^{T} \mathbf{D}_{-i}$, the Gram matrix is obtained by deleting the reading sensor $i$ which is most affected by interferences, and its signal-tonoise ratio which is below the admitted level specified by the system. In another words, the sensor reading having the lowest influence on the linear independence of column of the matrix $\mathbf{D}$ is deleted.

We propose an iterative procedure starting with a number of $M$ potential locations and ending with an optimum 
number of sensors $N$. By deleting the potential sensor location with the smallest contribution (maximum interference) the relative change in the determinant of the Gram matrix is the smallest, and so this method does provide a local maximization of the determinant of the Gram matrix. ${ }^{7}$ The first step in our iteration loop (see also Section 5) is to calculate (12) for all sensor locations, and thus the sensor corresponding to the lowest value of the determinant $1-d_{i}^{T} G_{-i}^{-1} d_{i}$ is removed. The resulting Gram matrix is updated with the reduced number of the remaining sensors in the system, and the above procedure is repeated until the number of sensors is reduced to the given number of sensors $N$.

The motivation for which the sensor locations are deleted in an iterative fashion, and not all at once, is twofold. First reason is due to the nature of interference location in signal space [14]. One might think that deleting more sensors for which the interference is higher is more appropriate. However, if the users are located in signal spaces, which are orthogonal to each other, then that number of users, which are experiencing higher interference, cannot have a higher contribution to the total interference in the system. This statement was proved in the context of CDMA systems in [16]. Secondly, in future wireless sensors networks, each sensor will try to adapt to the total interference in the system and will place itself in that region of signal space where there is minimum interference.

\section{SENSTIVITY ANALYSIS}

A survey of the literature shows that the sensitivity analysis for sensor location for systems identification has been investigated intensively $[4,11,23,24]$. In [24] the optimal sensor location for parameter identification of linear structural systems discusses the influence of noise on these. In [11] the effect of noise on sensor placement for on-orbital identification of large structures is studied, and it is suggested that erroneous estimates of the parameters can be ameliorated by placing the sensors at points of maximum sensitivity. However, very few papers have addressed the problems of sensitivity analysis for engine health monitoring.

Having identified sensor location, the problem is which criterion, among the combinations of all possible sensor locations, is to be adopted in order to select an optimal set effectively, such that the measured data will give desirable results for achieving a better estimation of structural states. We propose as a criterion the spectral condition number of Gram matrix $\mathbf{G}_{-i}=\mathbf{D}_{-i}^{T} \mathbf{D}_{-i}$ defined below:

$$
\lambda\left(\mathbf{G}_{-i}\right)=\left\|\mathbf{G}_{-i}\right\|\left\|\mathbf{G}_{-i}^{+}\right\|,
$$

where the superscript "+" denotes the pseudoinverse. The criterion is to locate sensors at those positions where the spectral condition number $\lambda\left(\mathbf{G}_{-i}\right)$ reaches its minimum, to achieve the best control effect. In this way, we determine

\footnotetext{
7 The determinant of Gram matrix can be also expressed in terms of its eigenvalues. The issue and the convergence of the global maximization of the eigenvalues of the Gram matrix were explored in [14].
}

the conditions under which the number of sensors will remain optimal when the data measured and used for sensitivity analysis are contaminated by additive noise (the same problem in the presence of colored noise is open and left for future research).

Assume that the measured responses given by (2) are corrupted in the presence of perturbation noise in an additive fashion:

$$
\tilde{\mathbf{y}}=\mathbf{y}+\boldsymbol{\mu}
$$

where $\boldsymbol{\mu}$ is the noise perturbation vector from the same Gaussian field. Correspondingly, the perturbed Gram matrix $\widetilde{\mathbf{G}}_{-i}$ can be expressed as

$$
\widetilde{\mathbf{G}}_{-i}=\mathbf{G}_{-i}+\boldsymbol{\eta}_{-i}
$$

where $\boldsymbol{\eta}_{-i}$ this time is a noise matrix whose properties related to the number of users communicating in the system are studied in [14]. Similar to [16] we assume in this paper that the signals and noises are statistically independent of each other. We can define the spectral condition number for the perturbed Gram matrix as $\lambda\left(\widetilde{\mathbf{G}}_{-i}\right)=\left\|\widetilde{\mathbf{G}}_{-i}\right\|\left\|\widetilde{\mathbf{G}}_{-i}^{+}\right\|$following (13). Using (15) and, based on (13), we obtain for the spectral number of perturbed Gram matrix the following expression:

$$
\lambda\left(\widetilde{\mathbf{G}}_{-i}\right)=\left\|\widetilde{\mathbf{G}}_{-i}\right\|\left\|\widetilde{\mathbf{G}}_{-i}^{+}\right\|=\left\|\mathbf{G}_{-i}+\boldsymbol{\eta}_{-i}\right\|\left\|\widetilde{\mathbf{G}}_{-i}^{+}\right\| .
$$

Using matrix perturbation theory [25] an upper perturbation bound for $\left\|\widetilde{\mathbf{G}}_{-i}^{+}\right\|$is given as

$$
\left\|\tilde{\mathbf{G}}_{-i}^{+}\right\| \leq\left(1+\frac{\alpha \beta}{1-\alpha}\right)\left\|\mathbf{G}_{-i}^{+}\right\|
$$

when $\alpha=\left\|\mathbf{G}_{-i}^{+}\right\|\left\|\boldsymbol{\eta}_{-i}\right\|<1$ and $\beta=(1+\sqrt{5}) / 2$. In order to obtain the dependence between the condition number $\lambda\left(\mathbf{G}_{-i}\right)$ of the initial Gram matrix in the absence of noise and the condition number $\lambda\left(\widetilde{\mathbf{G}}_{-i}\right)$ of the perturbed Gram matrix, we have to use the results from [25]. It was proved in [25] that when the rank of Gram matrix before and after perturbation is the same, then we obtain

$$
\left\|\mathbf{G}_{-i}+\boldsymbol{\eta}_{-i}\right\|=\gamma \lambda\left(\mathbf{G}_{-i}\right)
$$

where $0<\gamma \leq 1$. Substituting (18) and (17) in (16) we obtain the desired results:

$$
\lambda\left(\widetilde{\mathbf{G}}_{-i}\right)=\gamma\left(1+\frac{\alpha \beta}{1-\alpha}\right) \lambda\left(\mathbf{G}_{-i}\right)
$$

The fundamental result provided by (19) will allow us to check if the sensor locations determined in noise-free conditions are insensitive to AWGN under the proposed criterion above. In (19) we need $(1+\alpha \beta /(1-\alpha)) \rightarrow 1$, or a sufficient condition that $\alpha \rightarrow 0$. In other words, we need the norm of noise $\left\|\boldsymbol{\eta}_{-i}\right\| \rightarrow 0$ or small perturbation affecting the configuration of the initial number of sensors. This result is verified in our simulations. 


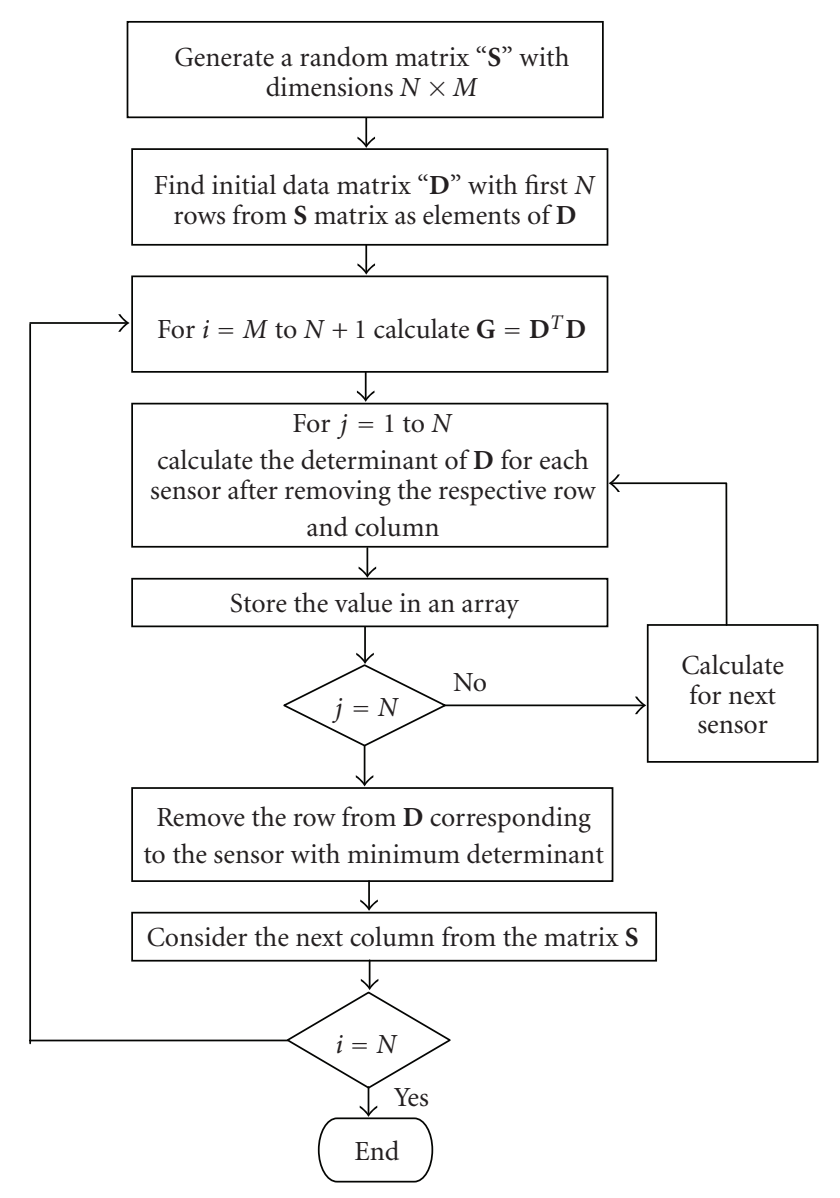

FIGURE 1: Overview of the methodology for maximizing the determinant of the Gram matrix.

\section{COMPUTATIONAL PROCEDURE FOR OPTIMAL SENSOR PLACEMENT METHOD}

Maximization of the determinant of the Gram matrix results in minimizing the error covariance matrix given by (4), which produces the best estimate of $\hat{q}$ in (3). Hence the sensors should be placed in a way that the determinant of the Fisher information matrix obtained as in (6) is maximized. When the procedure starts with $M$ sensor locations, it must be noted that the candidate sensor locations are deleted in an iterative fashion, and not all at once, because as the sensor locations which are the most affected by interferences are deleted, then a loss of linear independence is obtained. It is also important to note that when a sensor is deleted from the candidate sensor set, the total interference in the system decreases. The procedure is implemented using the algorithm described in Figure 1.

The implementation procedure for determining the optimal placement of sensors consists of the following steps.

(i) Generate first a random matrix $\mathbf{S}=\left[\mathbf{d}^{(1)}, \mathbf{d}^{(2)}, \ldots\right.$, $\left.\mathbf{d}^{(M)}\right]$ with dimensions $N \times M$. Algorithms to generate such matrices are given in MATLAB.

(ii) Generate a data matrix $\mathbf{D}$ of the designated pattern vectors $\mathbf{d}^{(i)}$ with dimension $N \times N$, where the ele-

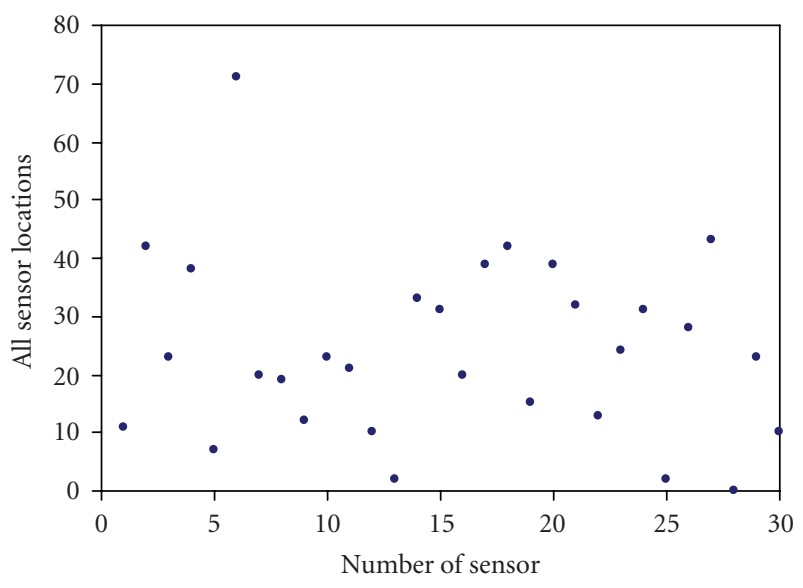

FIGURE 2: Randomly distribution of $M=30$ sensors before optimization.

ments of this data matrix $\mathbf{D}$ corresponds to the first $N$ columns in the matrix $\mathbf{S}$.

(iii) Find the Fisher information matrix associated with this matrix as in (6).

(iv) Calculate the determinant for the Gram matrix for each sensor using (12) for all sensors. This is done by deleting the rows and columns of matrix corresponding to the sensor reading with lowest interference (9), thus providing effective independence for the chosen sensor location.

(v) Remove the sensor which has least determinant from the data matrix by obtaining the matrix $\mathbf{D}_{-i}$.

(vi) Consider the next column $i, M-1 \geq i \geq N$, in $\mathbf{D}$ from the matrix $\mathbf{S}$ in order to update the Gram matrix $\mathrm{G}$ with a new sensor.

This procedure is repeated until an optimum matrix is obtained with all optimum sensor location set.

The performance of the above methodology depends on the measurements and, thus, the sensors used for engine health monitoring. Determination of the minimum number of sensors is associated with the cost of processing online, the initial placement of sensors, and other equipment criteria which are not considered here. The proposed methodology could be applied to any number of sensors $N \leq i \leq M$.

\section{NUMERICAL RESULTS}

The general computational procedure for optimal placement described in the previous section will be exemplified for a number of $M=30$ sensors which are contaminated by AWGN with a signal-to-noise ratio of $\beta=10 \mathrm{dBm}$, and which are randomly distributed ${ }^{8}$ on a rectangular area as in Figure 2. We consider the "normalized locations" in order to show the scalability of our method. Each sensor is affected

\footnotetext{
${ }^{8}$ It is well known that a sensor network can be deployed in two ways: random placement and grid placement. For an unknown environment with multipath interference, the random placement is the best choice.
} 
by interferences from the rest of sensors in the system and is identified by a number between 1 and 30 .

\subsection{Experiment 1: optimum sensor location}

We chose $P=6$ and a sample of the random matrix $\mathbf{D}$ for $N=6$ sensors, given below. The sensor readings are given in $\mathrm{dBm}$ and are column vectors of this matrix ${ }^{9}$ :

D

$$
=\left[\begin{array}{cccccc}
-0.3756 & -1.4048 & 0.7413 & 1.2376 & 0.2296 & -2.3779 \\
0.2077 & -1.0306 & 0.8120 & 1.2960 & 0.3553 & -1.0923 \\
-0.7656 & -0.6434 & -0.1428 & -0.2782 & 0.5213 & -0.3257 \\
-0.1064 & 0.1708 & -0.0999 & 0.2171 & -0.6160 & -2.0122 \\
0.3388 & 1.3448 & -0.8001 & 0.6307 & 1.3458 & 1.5677 \\
1.0335 & 1.9363 & 0.4932 & -0.5485 & 0.9749 & 0.2333
\end{array}\right] \text {. }
$$

We implemented a program in MATLAB, following the steps described in Section 5, eliminating the least effective sensor in each step of the iteration until 6 sensors were left. In each iterative step we found the corresponding Fisher information matrix. We eliminated one sensor at a time by deleting the corresponding row and column, and then optimizing the Gram determinant. We removed the sensor which produced the minimum Gram determinant value. The next iteration began and we ran the algorithm for $N \leq i \leq M$.

The procedure continued until the optimal placement of the final 6 sensors was obtained. After running the computational procedure described in Section 5, the optimal placement was obtained as in Figure 3 where only $N=6$ sensors are remained, namely, the sensors at locations 1, 2, 3, 16, 22, and 30 .

We noticed that the sensors, which are much close to each other, are eliminated from the initial assignment. The explanation for this is that the power received is inversely proportional to the distance from the transmitter. The remaining sensors experienced this lesser interference.

\subsection{Experiment 2: sensitivity analysis}

This time we considered a different number of sensors and the same data matrix as in Section 6.1. The optimal sensor placement was different and the results of the sensitivity analysis for $M=25, N=6, P=6, \sigma=1$, and sensors placed at locations 4, 6, 9, 16, 19, and 23 are represented in Figure 4.

One can observe that at these locations the condition number based on the Frobenius norm derived in Section 4 is minimum.

\footnotetext{
${ }^{9}$ In the context of engine health monitoring, we used real data from direct measurements. Even the matrix $\mathbf{D}$ is generated by simulation; in this experiment, the final sensor position is not dependent of realization of this matrix. In other words, the optimized location will remain the same if initial sensor readings are changed.
}

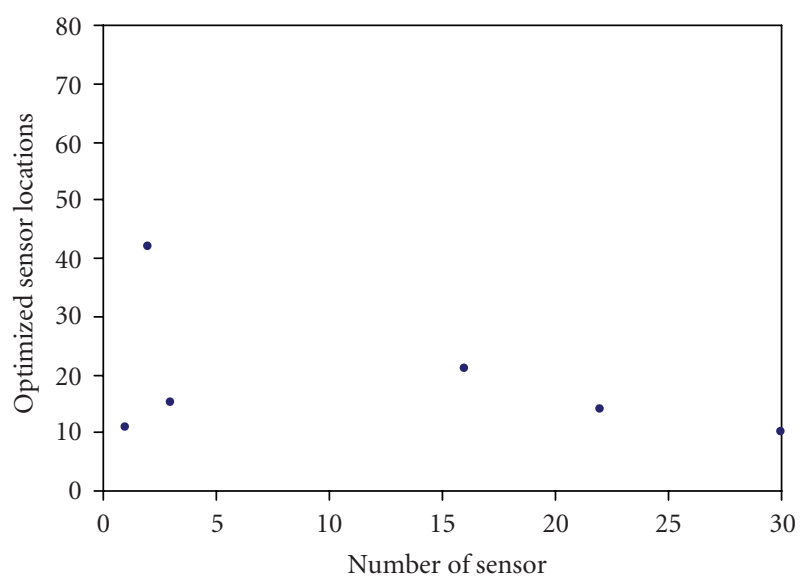

FIGURE 3: The optimum locations obtained with the proposed method.

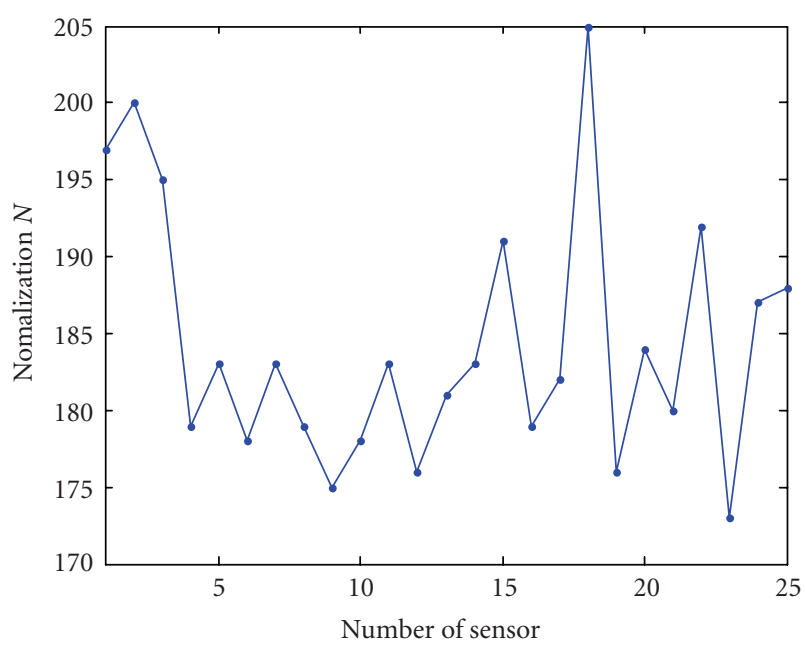

Figure 4: Sensitivity analysis for 25 sensors.

\subsection{Experiment 3: the influence of the noise on optimal placement}

It is sufficient to consider two optimal arbitrary locations $x$ and $y$, and the values of the two random estimates $q_{x}$ and $q_{y}$, respectively, along with the random noise vector $\mathbf{w}$, which are obtained with (2). In order to verify the robustness of the methodology proposed in Section 4, we needed to consider various levels of noise. For our purposes we considered the variance of the noise in (6) given by $\sigma_{w}^{2}=\left(\sigma_{q_{x}}^{2}+\sigma_{q_{y}}^{2}\right) \times \alpha$ where the parameter $\alpha \in[1,3]$.

We started this experiment with the same data matrix D as in Section 6.1 by using the algorithm presented in Figure 5 to calculate the mean variance and the covariance of random variables $q_{x}$ and $q_{y}$. As an illustrative example, we chose $x=1$ and $y=3$, and 100 values were generated for each random variable. The corresponding estimated data were $Q_{1}=\left[q_{1}^{(1)}, q_{1}^{(2)}, \ldots, q_{1}^{(100)}\right]$, and, respectively, 


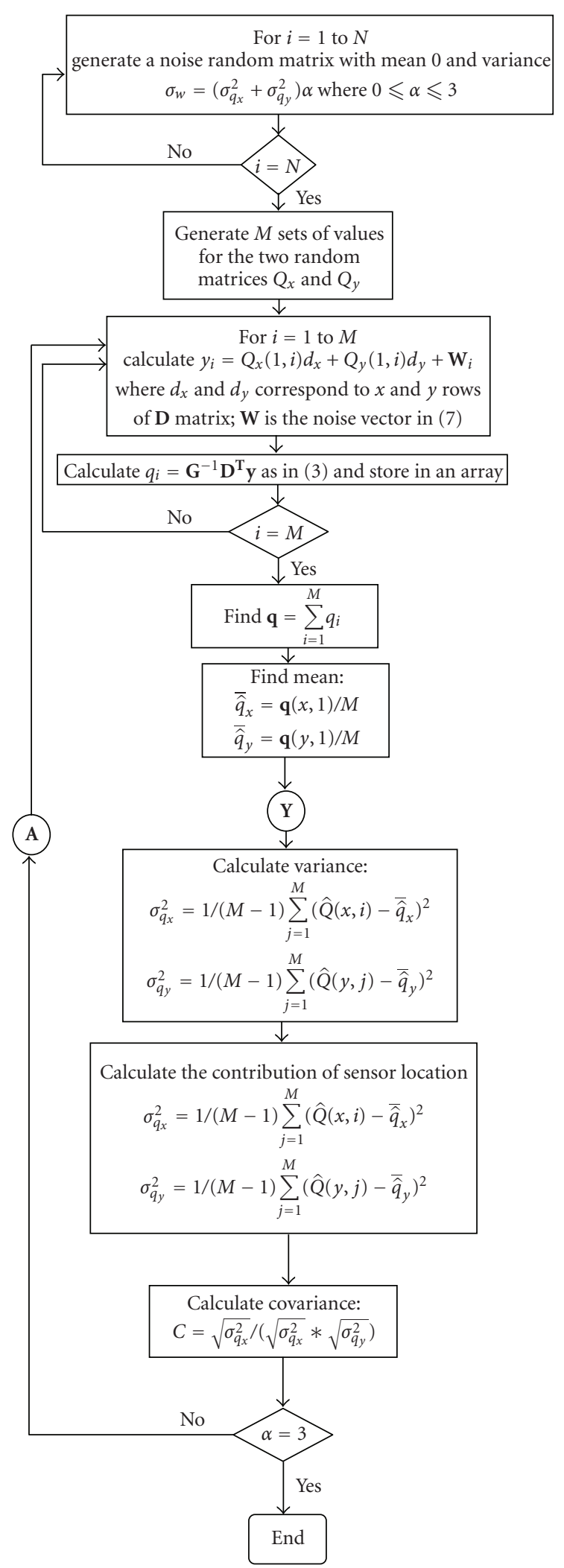

Figure 5: Overview of the diagram to generate the characteristics of the two random variables.

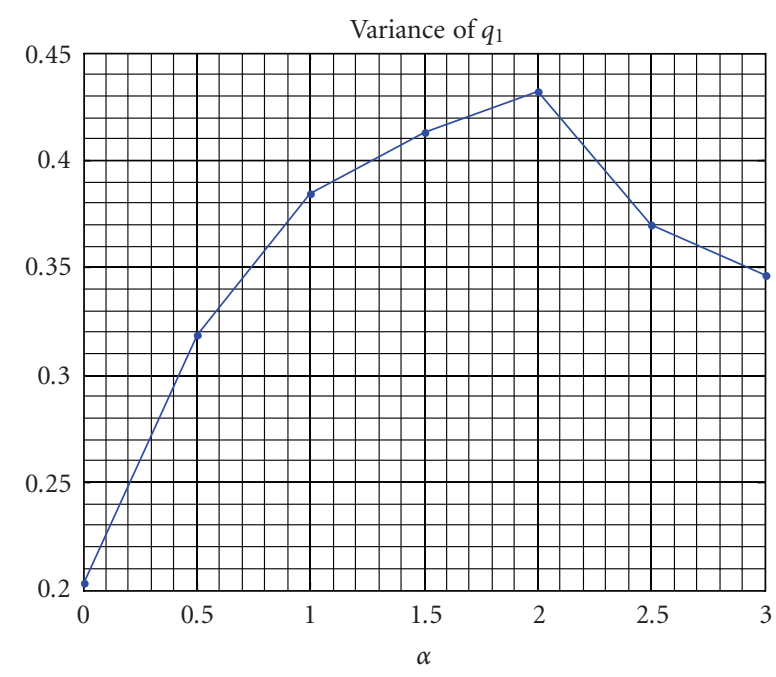

(a)

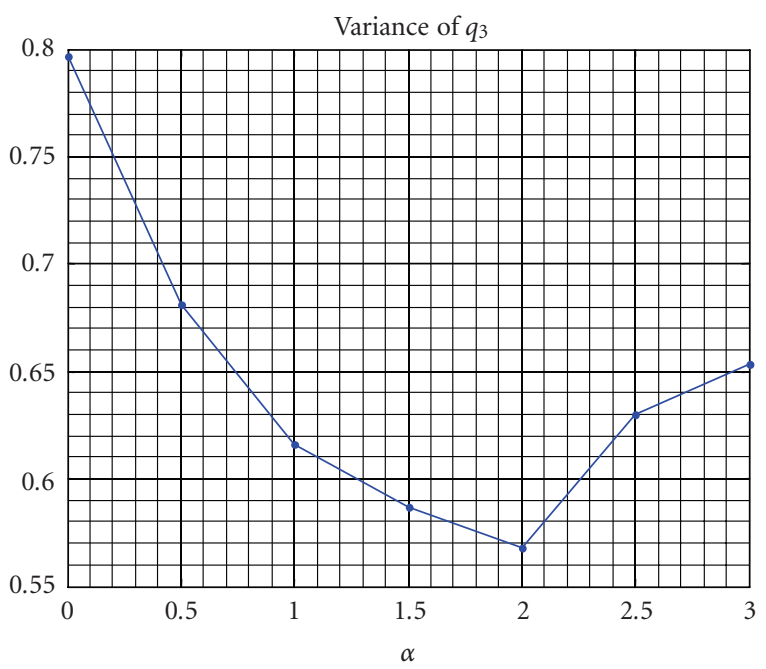

(b)

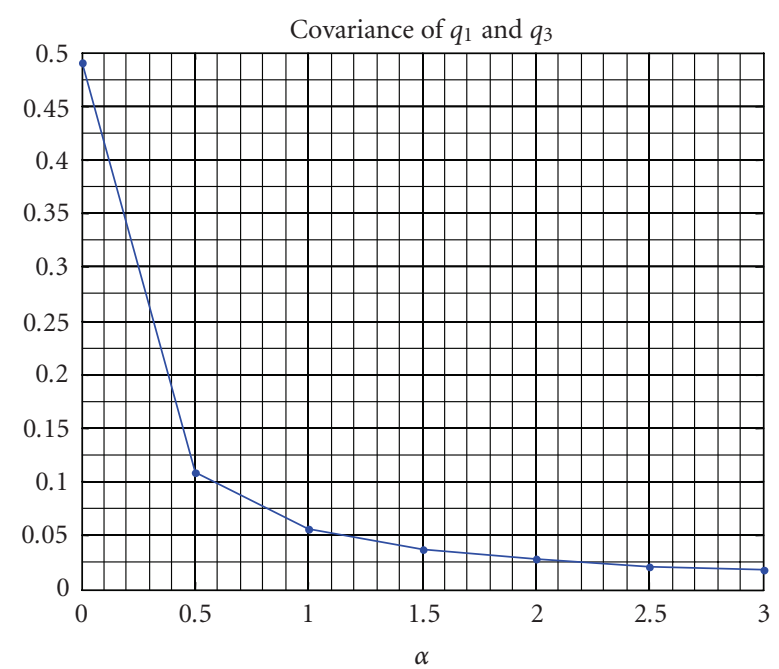

(c)

FIGURE 6: The influence of AWGN to the sensors located at optimal positions 1 and 3 . 
$Q_{3}=\left[q_{3}^{(1)}, q_{3}^{(2)}, \ldots, q_{3}^{(100)}\right]$. The mean of $\hat{Q}_{1}$ and $\hat{Q}_{3}$ was calculated by using the formulae

$$
E\left(\hat{Q}_{1}\right)=\frac{\sum_{i=1}^{100} \hat{q}_{i}}{100}=\overline{\hat{q}}_{1}, \quad E\left(\hat{Q}_{3}\right)=\frac{\sum_{i=1}^{100} \hat{q}_{i}}{100}=\overline{\hat{q}}_{3},
$$

the variances of $\hat{Q}_{1}$ and $\hat{Q}_{3}$ were calculated as follows:

$$
\begin{aligned}
& \operatorname{Var}\left(\hat{Q}_{1}\right)=\sigma_{\hat{q}_{1}}^{2}=\frac{1}{100-1} \sum_{j=1}^{100}\left(\hat{q}_{1}^{(j)}-\overline{\hat{q}}_{1}\right)^{2}, \\
& \operatorname{Var}\left(\hat{Q}_{3}\right)=\sigma_{\hat{q}_{3}}^{2}=\frac{1}{100-1} \sum_{j=1}^{100}\left(\hat{q}_{3}^{(j)}-\overline{\hat{q}}_{3}\right)^{2} .
\end{aligned}
$$

We used the following formula for their covariance: $\eta_{13}=$ $\sqrt{\sum_{j=1}^{100} \hat{q}_{1}^{(j)} \cdot \hat{q}_{3}^{(j)}} / \sqrt{\sum_{j=1}^{100}\left(\hat{q}_{1}^{(j)}\right)^{2} \sum_{j=1}^{100}\left(\hat{q}_{3}^{(j)}\right)^{2}}$.

The contribution of each linear receiver, $c_{i}, 1 \leq i \leq 6$ to the total signal-to-noise ratio in the system of $M$ sensors can be calculated individually as a proportion of the measurement data variation. With the values of the variances, the coefficients $q_{1}$ and $q_{3}$ in (3) or in (8) are calculated using the formulae $q_{1}=\sigma_{\hat{q}_{1}}^{2} /\left(\sigma_{\hat{q}_{1}}^{2}+\sigma_{\hat{q}_{3}}^{2}\right)$ and $q_{3}=\sigma_{\hat{q}_{3}}^{2} /\left(\sigma_{\hat{q}_{1}}^{2}+\sigma_{\hat{q}_{3}}^{2}\right)$, respectively. Their variances are obtained for varying values of $\alpha$ in the interval $0 \leq \alpha \leq 3$, and are plotted in Figures 6(a) and $6(\mathrm{~b})$.

It is verified in this simulation that for these coefficients the sum of variances at these locations is always constant. As the noise level increases, their values increase or decrease toward their asymptotic values, 0.34632 and 0.65368 , respectively. It is useful to analyze the correlation coefficient for these two optimal locations for $0 \leq \alpha \leq 3$. For a small number of generated samples, the correlation coefficient is not zero (0.0188), as can be observed from Figure 6(c). However, we should expect, in general, the measurements to be statistically independent.

The influence of the noise AWGN for all $N=6$ sensors in the optimal placement of Section 6.1 is represented in Figure 7. If the level of noise increases, then the values of sensor readings are affected in the same way. However, for the sensor placed at location 30, the value of the sensor reading is not affected, as this sensor is far away from the others. According to [14] this sensor is oversized and is the most robust to noise interference.

In Figure 8, we varied the number of sensors and considered the same characteristics of the noise. We noticed that more sensors provided more information, and the proposed method seems to gain robustness with the number of sensors. By increasing the number of sensors, which are optimally distributed, the readings in the presence of the noise are more robust (stronger signal) and the effect of random noise may be suppressed more effectively.

\section{CONCLUSIONS}

In this paper, we analyzed the problem of optimal placement of sensors in the presence of AWGN considering the sensor as a system having full communication capabilities and using minimum interference algorithms. We used a Gram matrix

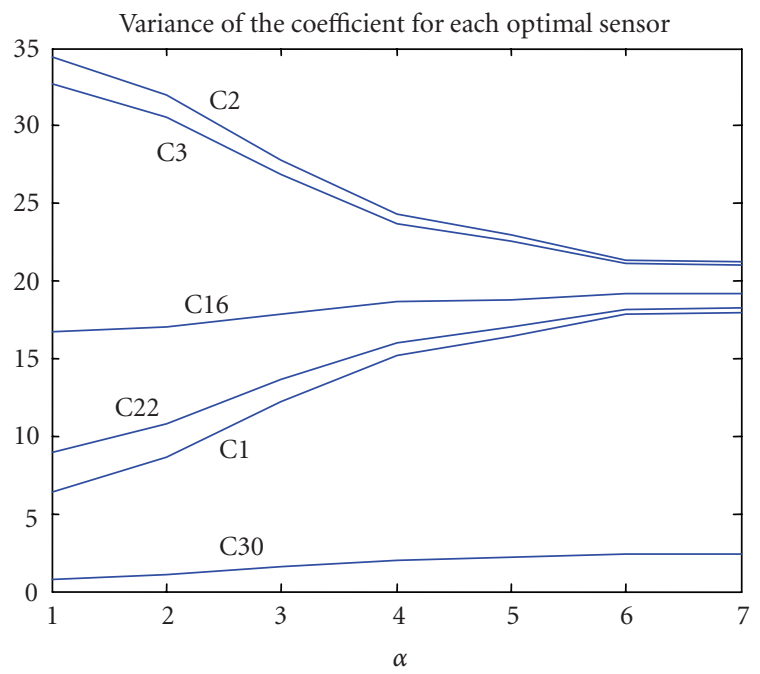

FIGURE 7: Variation of the sensor readings in the presence of AWGN.

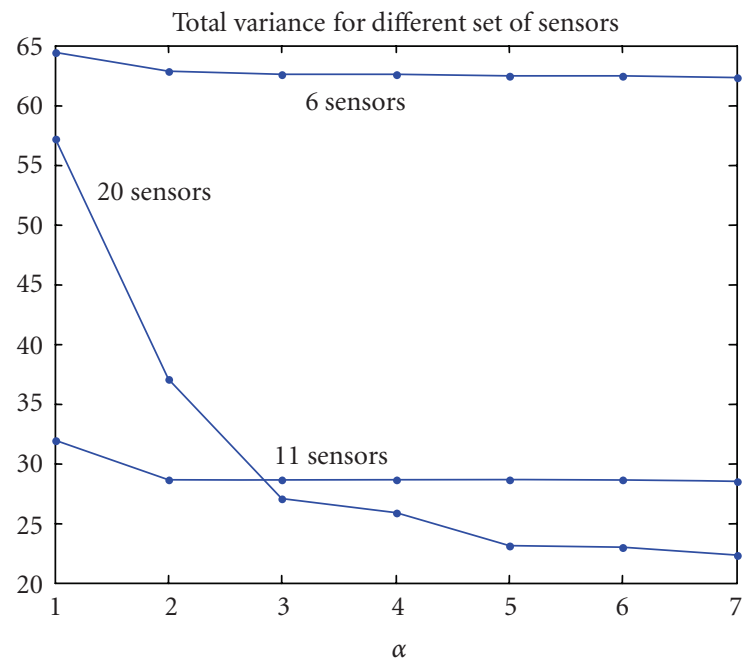

FIGURE 8: Influence of the noise on different number of sensors optimally distributed.

approach for the sensitivity analysis and our simulations validated the mathematical formulation. The proposed method is robust, and the influence of noise is minimized as the number of sensors increases.

The optimization problem presented in this paper was solved considering the same requirement in terms of signalto-interference ratio for each sensor. We provided detailed numerical examples to support our mathematical formulation. Our future research will focus on the extension of our results to different classes of sensors having the same signalto-interference ratio and in the presence of colored noise.

\section{ACKNOWLEDGMENTS}

The authors thank the Associate Editor, Professor Erchin Serpedin, and the anonymous reviewers for helpful suggestions that improved the quality of this paper. This work was 
supported in part by Air Force Office of Scientific Research Agency under Project no. FA9950-04-1-0254 and NSF Grant no. IIP 0733885. This paper was presented in part at the IEEE International Conference on System of Systems Engineering, IEEE SoSE 2007, April 16-18, San Antonio, TX, USA, 2007.

\section{REFERENCES}

[1] Q. Rong, D. Ceglarek, and J. Shi, "Dimensional fault diagnosis for compliant beam structure assemblies," Journal of Manufacturing Science and Engineering, vol. 122, no. 4, pp. 773-780, 2000.

[2] Y. Ding, P. Kim, D. Ceglarek, and J. Jin, "Optimal sensor distribution for variation diagnosis in multistation assembly processes," IEEE Transactions on Robotics and Automation, vol. 19, no. 4, pp. 543-556, 2003.

[3] Y. Wang and S. R. Nagarkar, "Locator and sensor placement for automated coordinate checking fixtures," Journal of Manufacturing Science and Engineering, vol. 121, no. 4, pp. 709-719, 1999.

[4] Y. Y. Li and L. H. Yam, "Sensitivity analyses of sensor locations for vibration control and damage detection of thin-plate systems," Journal of Sound and Vibration, vol. 240, no. 4, pp. 623-636, 2001.

[5] M. Basseville, A. Benveniste, G. V. Moustakides, and A. Rougee, "Optimal sensor location for detecting changes in dynamical behavior," IEEE Transactions on Automatic Control, vol. 32, no. 12, pp. 1067-1075, 1987.

[6] A. Arbel, "Sensor placement in optimal filtering and smoothing problems," IEEE Transactions on Automatic Control, vol. 27, no. 1, pp. 94-98, 1982.

[7] R. K. Mehra, "Optimization of measurement schedules and sensor design for linear dynamic systems," IEEE Transactions on Automatic Control, vol. 21, no. 1, pp. 55-64, 1976.

[8] R. Stolkin, L. Vickers, and J. V. Nickerson, "Using environmental models to optimize sensor placement," IEEE Sensors Journal, vol. 7, no. 3, pp. 319-320, 2007.

[9] A. Khan, D. Ceglarek, J. Shi, J. Ni, and T. C. Woo, "Sensor optimization for fault diagnosis in single fixture systems: a methodology," Journal of Manufacturing Science and Engineering, vol. 121, no. 1, pp. 109-117, 1999.

[10] D. Ceglarek and J. Shi, "Fixture failure diagnosis for auto body assembly using pattern recognition," Journal of Engineering for Industry, vol. 118, pp. 55-66, 1996.

[11] D. C. Kammer, "Optimal sensor placement for modal identification using system-realization methods," Journal of Guidance, Control, and Dynamics, vol. 19, no. 3, pp. 729-731, 1996.

[12] G. C. Franchi and G. Gallieni, "Genetic-algorithm-based procedure for pretest analysis," AIAA Journal, vol. 33, no. 7, pp. 1362-1364, 1995.

[13] H. Y. Guo, L. Zhang, L. L. Zhang, and J. X. Zhou, "Optimal placement of sensors for structural health monitoring using improved genetic algorithms," Smart Materials and Structures, vol. 13, no. 3, pp. 528-534, 2004.

[14] P. Cotae, "Transmitter adaptation algorithm for multicellular synchronous DS-CDMA systems with multipath," IEEE Journal on Selected Areas in Communications, vol. 24, no. 1, pp. 94-103, 2006.

[15] P. Cotae, "Multicell spreading sequence design algorithm for overloaded S-CDMA," IEEE Communications Letters, vol. 9, no. 12, pp. 1028-1030, 2005.

[16] P. Cotae, "On the optimal sequences and total weighted square correlation of synchronous CDMA systems in multipath chan- nels," IEEE Transactions on Vehicular Technology, vol. 56, no. 4, pp. 2063-2072, 2007.

[17] L. Yao, A. W. Sethares, and C. D. Kammer, "Sensor placement for on-orbit modal identification via a genetic algorithm," AIAA Journal, vol. 31, no. 10, pp. 1922-1928, 1993.

[18] D. Ceglarek and J. Shi, "Dimensional variation reduction for automotive body assembly," Manufacturing Review, vol. 8, no. 2, pp. 139-154, 1995.

[19] D. Djurdjanovic and J. Ni, "Stream of variation based analysis and synthesis of measurement schemes in multi-station machining systems," in Proceedings of the ASME International Mechanical Engineering Congress and Exposition, vol. 12, pp. 297-304, New York, NY, USA, November 2001.

[20] L. W. Poston and H. R. Tolson, "Maximizing the determinant of the information matrix with the effective independence method," Journal of Guidance, Control, and Dynamics, vol. 15, no. 6, pp. 1513-1514, 1992.

[21] Q. Rong, D. Ceglarek, and J. Shi, "Adjusted least squares approach for diagnosis of ill-conditioned compliant assemblies," Journal of Manufacturing Science and Engineering, vol. 123, no. 3, pp. 453-461, 2001.

[22] G. Strang, Linear Algebra and Its Applications, Harcourt Brace, Orlando, Fla, USA, 3rd edition, 1988.

[23] M. Chang and D. C. Gossard, "Computational method for diagnosis of variation-related assembly problems," International Journal of Production Research, vol. 36, no. 11, pp. 2985-2995, 1998.

[24] P. H. Kirkeaard and R. Brincker, "On the optimal location of sensors for parametric identification of linear structural systems," Mechanical Systems and Signal Processing, vol. 8, no. 4, pp. 639-647, 1994.

[25] W. Stewart and J.-G. Sun, Matrix Perturbation Theory, Academic Press, Boston, Mass, USA, 1990. 